\title{
Neue Tendenzen in der bilateralen schweizerischen Entwicklungszusammenarbeit
}

Rudolf Dannecker

\section{(2) OpenEdition}

Édition électronique

URL : http://journals.openedition.org/sjep/1420

DOI : 10.4000/sjep. 1420

ISSN : 1663-9677

Éditeur

Institut de hautes études internationales et du développement

\section{Édition imprimée}

Date de publication : 1 janvier 1994

Pagination : 237-250

ISSN : 1660-5926

\section{Référence électronique}

Rudolf Dannecker, « Neue Tendenzen in der bilateralen schweizerischen

Entwicklungszusammenarbeit », Schweizerisches Jahrbuch für Entwicklungspolitik [En ligne], 13 | 1994 mis en ligne le 04 juin 2013, consulté le 08 septembre 2020. URL : http://journals.openedition.org/ sjep/1420; DOI : https://doi.org/10.4000/sjep.1420 


\title{
Neue Tendenzen in der bilateralen schweizerischen Entwicklungszusammenarbeit
}

\author{
Rudolf Dannecker
}

\section{Das veränderte internationale und nationale Umfeld}

Die Veränderung der weltpolitischen Machtkonstellation in den letzten Jahren hat die internationale Entwicklungszusammenarbeit entscheidend geprägt; sie hat zu massgeblichen Veränderungen und zu einer Verlagerung von Schwerpunkten geführt. Der Fall der Berliner Mauer und die anschliessenden politischen Umwälzungen mit dem Zusammenbruch des kommunistischen Systems im Osten haben sowohl auf der Geber- wie auch auf der Empfängerseite der Hilfe neue Entwicklungen gefördert. So hat u.a. das Ende des "Kalten Krieges" die Möglichkeiten der Entwicklungsländer stark eingeschränkt, sich ihr aussenpolitisches Wohlverhalten und die Zugehörigkeit zu einem der beiden Blöcke durch wirtschaftliche Unterstützung und Entwicklungshilfe vergüten zu lassen. Auf der Geberseite stiegen dagegen die Anforderungen an die Empfänger der Hilfe, da aussenpolitische oder militärische Auflagen vermehrt durch wirtschaftliche und innenpolitische Konditionalitäten abgelöst wurden. Es ist uns wieder bewusster geworden, dass in der Vergangenheit neben anderen Motiven auch der Ost-West-Gegensatz mit ein Grund für Entwicklungshilfeleistungen war. Deutlich wird dies an der Verlagerung nicht nur des politischen Interesses, sondern auch von finanziellen Ressourcen vom Süden nach dem Osten, am Rückgang der Hilfeleistungen sowie an Verlagerungen von der Langfristhilfe zu Massnahmen der Friedenssicherung und der Nothilfe. Verschärft wird diese Situation durch eine Reihe weiterer Faktoren. Die tiefgreifende wirtschaftliche Rezession hat in vielen Staaten zu massiven Ver- 
schuldungen der öffentlichen Haushalte und in der Folge zu entsprechenden Budgetkürzungen geführt. Die Budgets für internationale Zusammenarbeit sind bei diesen internen Budgetverteilungs-Auseinandersetzungen besonders gefährdet, da sie nicht über einflussreiche Lobbies verfügen und Kürzungen innenpolitisch auf weniger politischen Widerstand stossen als andere Bereiche. Gleichzeitig steigen, aufgrund zunehmender bürgerkriegsähnlicher Situationen in verschiedenen Teilen der Welt, die Ausgaben für friedenssichernde Massnahmen und humanitäre Hilfe. Insbesondere Friedenssicherung ist oft mit sehr hohen finanziellen Kosten verbunden.

Die Beendigung des "Kalten Krieges" hat auch in Entwicklungsländern tiefgreifende Auswirkungen: Der Wegfall der politischen Blöcke verändert die innenpolitischen Kräfteverhältnisse und führt zu einer grösseren Pluralität und zu einer politisch sensibilisierteren Bevölkerung. Dies wiederum hat - nach Jahrzehnten von festzementierten, autoritären Regierungen - in einer ersten Phase innenpolitische Umwälzungen und Unsicherheiten zur Folge, wie sie für Übergangszeiten unvermeidlich sind. Erfolgreiche Anpassungen und Reformen benötigen aber auch finanzielle Unterstützung. Der sogenannte Demokratiebonus, d.h. die besondere Honorierung derjenigen Länder, die auf dem Weg der Demokratisierung besonders aktiv voranschritten, ist von der internationalen Hilfe bisher kaum eingelöst worden. Es ist bedauerlich, dass gerade jetzt Mittel fehlen, um den reformorientierten Kreisen den Rücken zu stärken, nachdem Geberländer während Jahren regelmässig bessere und an einer langfristigen Entwicklungspolitik orientierte Verwaltungen und Regierungen gefordert haben.

Zusätzlich zum schwierigen Übergang von autoritär geprägten Regierungen und Verwaltungssystemen zu pluralistischen Formen sehen sich viele Entwicklungsländer mit tiefen Preisen der von innen exportierten Rohstoffe auf den Weltmärkten konfrontiert. Dies trägt - zusammen mit der Verschuldung und einer oft nicht angepassten Wirtschaftspolitik - dazu bei, dass der Staat, insbesondere in Afrika, nicht mehr in der Lage ist, die finanziellen Aufwendungen für zentrale staatliche Aufgaben, wie beispielsweise Volksschulen, Gesundheitsdienste oder andere wichtige staatliche Dienstleistungen, zu erbringen.

Vor diesem Hintergrund der Rezession, des weltpolitischen Umbruchs, der Herausbildung von neuen Kräftezentren politischer und wirtschaftlicher Macht, der verstärkten Orientierung der europäischen Interessen in Richtung Osteuropa sowie der innenpolitischen Umbruchssituationen und Strukturanpassungen wird auch die Frage nach den Resultaten der bisherigen Hilfe gestellt. Die Zeit der 60er Jahre ist vorbei, in der die Hilfe an den Süden als einmalige Anstrengung einer Generation, als Technik- und Ressourcentransfer, als geschickter Mix von verschiedenen Inputs gesehen wurde. War man damals rasch mit "Patentmitteln" und einfachen Wunderlösungen zur Hand, so breitet sich heute in der Öffentlichkeit eine gewisse Müdigkeit des Helfens aus. Die Zeit breit abgestützter SympathieAllianzen für die Entwicklungszusammenarbeit ist vorbei. Vor dem Hintergrund interner Probleme im Sozialbereich und dem Überschwappen von Drittweltproblemen, wie z.B. Migrationen und Asylfragen, auf unsere Innenpolitik, nimmt die internationale Solidarität mit dem Süden ab. 
Gleichzeitig erleben wir in der Schweiz schmerzhafte Strukturanpassungen in einer rasch sich globalisierenden Welt, und dies in den verschiedensten Lebensbereichen. In dieser unruhigen Zeit der Veränderung ist gerade die sich an Ruhe und langsames, pragmatisches Entwickeln gewohnte Schweiz besonderen Herausforderungen ausgesetzt, wenn es gilt, sich mit weltweiten, grundlegenden Veränderungen und dem Strukturwandel im eigenen Land auseinanderzusetzen. Welches sind in dieser neuen Situation die Auswirkungen auf die schweizerische Entwicklungszusammenarbeit?

\section{Die Auswirkungen der Budgetrestriktionen auf die schweizerische Entwicklungszusammenarbeit: Die konkreten Foigen werden spürbar}

Was bedeuten - vor dem beschriebenen Hintergrund - die Budgetrestriktionen für die internationale Entwicklungszusammenarbeit und humanitäre Hilfe der Schweiz?

Vorerst ist es wichtig, die Mechanismen im Bereich der langfristigen Zusammenarbeit zu kennen. Im Gegensatz zur humanitären Hilfe und insbesondere zur Nothilfe, die im finanziellen Bereich ihre Ausgaben kurzfristig tätigen, sind in der Entwicklungszusammenarbeit die Ausgaben langfristig geplant und festgelegt, d.h. auf die nächsten 2-4 Jahre hinaus. Bereits heute müssen operationelle Entscheide getroffen werden, die im Verlaufe von 1995 - 1997 zu Auszahlungen führen. Als Orientierungsrahmen dient dabei der langfristige Finanzplan des Bundes und die vom Parlament genehmigten 4-Jahres-Verpflichtungskredite. Die effektiven Auszahlungsbudgets werden demgegenüber erst aufgrund der Budgetbeschlüsse des Parlaments festgelegt und sind jeweils im Dezember für das nächste Jahr bekannt. Die DEH muss somit auf verschiedenen Ebenen planen: Einerseits auf einer langfristigen Verpflichtungsebene (mit Rahmenkrediten des Bundes als Orientierung), andererseits auf der kurz- bis mittelfristigen Zahlungsebene mit den voraussichtlich zu erwartenden Jahresbudgets und kurzfristig mit den effektiv bewilligten Budgets. Deshalb können auch Kürzungen im Finanzplan erst mit einiger zeitlicher Verspätung umgesetzt werden. Gerade weil unsere Massnahmen auf 10-20 Jahre Dauer ausgerichtet sind und den Aufbau von neuen oder die Veränderung von bestehenden Strukturen zum Ziel haben, können Büdgetkürzungen die Anstrengungen früherer Jahre in Frage stellen. Sie können das Erreichen von bisherigen Projektzielsetzungen verunmöglichen und auch die Zusammenarbeitsbasis mit dem Vertragspartner in der Dritten Welt gefährden. Verschärft wird das Problem, da aus verschiedenen Gründen (insbesondere aufgrund von langfristigen vertraglichen Verpflichtungen) nicht überall gestrichen werden kann und Kürzungen umso stärker auf eine begrenzte Zahl von Programmen voll durchschlagen.

Was heissen diese Budgetrestriktionen nun ganz konkret für die DEH und ihr operationelles langfristiges Unterstützungsprogramm? Wie wird in dieser Situation vorgegangen, nach welchen Kriterien werden Kürzungsentscheide getroffen, wie Prioritäten gesetzt? 
- In einem ersten Schritt wurden die Programm- und Projektbudgets auf das für die Durchführung als notwendig erachtete Minimum linear gekürzt. In vielen Fällen hatte dies konkret zur Folge, dass die vorgesehenen Programme reduziert oder Teile davon von unseren Partnern aufgegeben werden mussten oder nicht in Angriff genommen werden konnten.

- In einem zweiten Schritt mussten neue Projektideen zurückgestellt oder aufgegeben werden. Da die Identifikation, Vorbereitung und Planung eines Projekts immer zusammen mit dem Partner erfolgt und längere Zeit dauert, bedeutet diese Vorbereitung, die für eine spätere gute Zusammenarbeit bei der Durchführung ausserordentlich wichtig ist, meistens einen hohen Zeitaufwand und eine beträchtliche Investition in die Zukunft.

- Anschliessend mussten Programme, die sich bereits in einem fortgeschrittenen Planungs- und Vorbereitungsstadium befanden, sistiert werden, wobei es sich sowohl um neue wie auch um die Erweiterung bestehender Programme handelte. Da wir einen pragmatischen Aufbau verfolgen und aufgrund konkreter Erfahrungen Programme schrittweise auf- und ausbauen, bedeuten Budgetkürzungen in solchen Fällen oft den Unterbruch eines organischen Wachstumprozesses einer bisher erfolgreichen Unterstützung. Ebenso mussten bereits in der Übergabephase stehende Projekte früher als geplant übergeben werden.

- Schliesslich mussten auch laufende Projekte und Programme beendet werden, da die zur Verfügung stehenden Budgetmittel nicht mehr zur Finanzierung aller laufenden Aktionen ausreichten. Konkret mussten beispielsweise die Ausbildung von Elektro- und Maschinentechnikern in Madagaskar und ein bereits im Detail vorbereitetes Programm für die Ausbildung von Instrumenten- und Gerätemechanikern im südlichen Afrika eingestellt werden. In Lateinamerika wurden Unterstützungsmassnahmen in der ländlichen Entwicklung, der Gewerbeförderung und der sozialen Infrastruktur zurückgestellt. In Indonesien musste die Mitfinanzierung eines UNICEF-Programms für die Gesundheit und Ernährung von Kleinkindern eingestellt werden. Die Zusammenarbeit mit zwei lokalen privaten Hilfswerken im Bereich der Basisgesundheit und Förderungsprogramme für Ureinwohner Indiens mussten aufgegeben werden. Diese kurze Aufzählung zeigt die konkreten Folgen der Budgetrestriktionen, wobei wir uns im wesentlichen von den folgenden Kriterien leiten lassen:

- Der bisherige Erfolg des in Frage stehenden Programms, insbesondere unter dem Aspekt der Nachhaltigkeit; d.h. die langfristigen Erfolgschancen des Programms ohne Unterstützung von aussen.

- Die Integration einer Unterstützungsmassnahme in ein grösseres Programm (sektorielle und institutionelle Schwerpunktbildung).

- Die geografische Schwerpunktbildung in einem Land, einer Region oder einer ökologischen Zone; d.h. vor allem Aufgabe von isolierten Einzelprojekten.

- Die Relevanz der Programme in bezug auf die Prioritäten des DEHLeitbildes. 
- Die Auswirkung von Kürzungen auf die Betroffenen (Bevölkerung etc.) sowie auf die Beziehungen zu unseren Partnern in Entwicklungsländern und in der Schweiz.

Budgetengpässe führen unvermeidlich zur Hinterfragung der Richtigkeit der gewählten Prioritäten, zu Konkurrenzsituationen zwischen Mitarbeiterinnen und Mitarbeitern, aber auch zwischen Sektionen und Diensten, zwischen verschiedenen Hilfskategorien. Dies war und ist auch in der DEH der Fall und hat neben vielen Schwierigkeiten und negativen Folgen auch längerfristige Konsequenzen. Bisherige Praktiken müssen hinterfragt, Konzepte in Frage gestellt werden. Programme müssen mit kleinen finanziellen Mitteln realisiert und angepasst und die zukünftige Ausrichtung des Programms ganz allgemein überdacht werden.

\section{Der Beitritt der Schweiz zu den Institutionen von Bretton Woods (BWI)}

Gleichzeitig mit den sich abzeichnenden Budgetproblemen erfolgte 1992 nach einer Volksabstimmung der Beitritt der Schweiz zur Weltbank und zum Internationalen Währungsfonds (IWF). Der Abstimmungskampf führte zu einer intensiven innenpolitischen Diskussion über die Ausrichtung der schweizerischen Entwicklungspolitik. Während eine Reihe von grossen schweizerischen Entwicklungsorganisationen den Beitritt unterstützten (wenn auch mit Forderungen für eine zukünftige aktive Rolle der Schweiz in diesen Organisationen), sprachen sich eine Reihe von kleineren Hilfswerken vor allem gegen den Beitritt zum IWF aus. Bedeutsam war, dass in einer so wichtigen aussenpolitischen Frage Regierung und Verwaltung einerseits sowie Hilfswerke als Teile der "Zivilgesellschaft" andererseits trotz zum Teil verschiedener Auffassungen in der Entwicklungspolitik bereit waren, in dieser Frage das gleiche übergeordnete Ziel zu verfolgen.

Für die schweizerische Entwicklungszusammenarbeit und damit für die $\mathrm{DEH}$ und das Bundesamt für Aussenwirtschaft BAWI (innerhalb der Verwaltung für die Beziehungen zur Weltbank federführend) ergaben sich durch den Beitritt zur Weltbank eine Reihe von Konsequenzen (dasselbe gilt sinngemäss für den IWF):

- Auf der Ebene der Mitwirkung in der Bank (Politik, Programme, Konzepte etc.) über den Exekutivdirektor der von der Schweiz angeführten Stimmrechtsgruppe.

- In der Zusammenarbeit im operationellen Projekt- und Programmbereich.

- Durch den innenpolitischen Dialog zwischen Verwaltung und insbesondere NGOs über die Rolle der Schweiz in der Weltbank.

- Durch die Führung einer Stimmrechtsgruppe in der Bank mit Polen und den vier zentralasiatischen Republiken Aserbaidschan, Kirgisistan, Turkmenistan und Usbekistan.

- Durch erhöhte finanzielle Leistungen innerhalb der schweizerischen Entwicklungszusammenarbeit an die Weltbankgruppe. 
Auf der Mitwirkungsebene im Verwaltungsrat der Weltbank haben die ersten Erfahrungen gezeigt, dass es auch einem kleinen Land wie der Schweiz möglich ist, über den Sitz im Exekutivrat einen nicht zu unterschätzenden Einfluss auszuüben. Um den Exekutivdirektor möglichst konkret von der schweizerischen bilateralen Erfahrung bei der Behandlung von Sachgeschäften, insbesondere von wichtigen Politikentscheiden, Landesprogrammen der Bank (Country Assistance Strategies) und Sektorprogrammen und -politiken profitieren zu lassen, ist eine enge Zusammenarbeit mit den Koordinationsbüros der DEH und den schweizerischen Botschaften in Entwicklungsländern organisiert worden. Dieser enge Austausch zwischen unserer Vertretung bei der Weltbank und den Koordinationsbüros ist uns aus zwei Gründen ausserordentlich wichtig:

- Einmal wird dadurch unsere Vertretung bei der Bank mit konkreten Informationen aus dem Feld und mit Beurteilungen der Weltbankarbeit aus bilateraler Sicht beliefert.

- Andererseits erfordert dies von unseren bilateralen Büros eine Auseinandersetzung mit dem Programm der Bank.

Im direkten Projekt- und Programmbereich werden die bisherigen Kofinanzierungsprogramme in Zukunft durch unsere Gesamtbeiträge an die IDA ersetzt. Gleichzeitig werden wir aber weiterhin direkte Kofinanzierungen durchführen, und dies vor allem dort, wo wir gegenseitige Synergien erwarten. Deshalb werden Kofinanzierungen auf Schwerpunktländer und -sektoren der DEH konzentriert, wo wir aufgrund unserer konkreten lokalen und sektoriellen Erfahrungen, unserer spezifischen Kenntnisse lokaler Verhältnisse sowie der Verhaltens- und Vorgehensweisen (z.B. Partizipation der Bevölkerung etc.) eine kompetente und gleichwertige Partnerin der Weltbank sind.

Im innenpolitischen Bereich - zwischen Verwaltung und schweizerischen Hilfswerken - hat die Diskussion um den Weltbankbeitritt aus Sicht der DEH zu einer Verstärkung der gegenseitigen Beziehungen geführt, zu einem intensiveren Informationsaustausch und zu einem engagierteren Dialog über unsere Rolle und unsere Politik in den BWI. Beide Seiten suchen dabei aktiv das Gespräch und die Chance zu einem vertieften Dialog zwischen Verwaltung und Hilfswerken wird genutzt.

Die Führung der Stimmrechtsgruppe im Exekutivrat, mit Polen und den zentralasiatischen Republiken, führt dazu, dass sich die Schweiz auch mit den Interessen dieser Staaten befasst. Interessanterweise sind es eine Reihe schweizerischer Hilfswerke, welche die Initiative ergriffen haben und gemeinsam Abklärungen über mögliche Unterstützungsmassnahmen unternehmen. Längerfristig dürfte es aber auch im Interesse der Schweiz liegen, ein bilaterales Zusammenarbeitsprogramm mit diesen Ländern aufzubauen.

Führt der Beitritt der Schweiz zur Weltbank zu einer Verminderung der schweizerischen bilateralen Hilfe und zu einer Steigerung des multilateralen Anteils? Während der Abstimmungskampagne hat der Bundesrat zugesichert, dass der Beitritt durch zusätzliche Mittel finanziert und nicht auf Kosten der bilateralen 
Unterstützugsmassnahmen erfolgen wird. Diese Verpflichtung ist auch mit einer separaten Budgetrubrik für die Beitrittskosten für die Periode 1992 - 1996 eingehalten worden. Mittelfristig allerdings, mit den steigenden Beiträgen an die IDA und mit weiteren Kürzungen, insbesondere auch durch das Parlament, könnte sich der Druck auf die bilaterale Hilfe der Schweiz verstärken. Allerdings ist offen, wo Kompensationen erfolgen, da neben der bilateralen technischen Zusammenarbeit und Finanzhilfe auch die humanitäre Hilfe, die wirtschafts- und handelspolitischen Massnahmen und die Entschuldungsmassnahmen des BAWI sowie die bilateralen Massnahmen im globalen Umweltschutzbereich Teil der gesamten bilateralen Massnahmen sind. Ebenso könnte auch das Parlament in den kommenden Budgetdiskussionen noch eigene Akzente setzen. Klar ist, dass bei zukünftigen Budgetauseinandersetzungen die Frage nach den Anteilen der verschiedenen Kategorien der öffentlichen Hilfe gestellt wird.

Der Beitritt der Schweiz zur Weltbank bildet somit für die schweizerische Entwicklungszusammenarbeit ein Potential, dass sie nutzen kann. Es ist unser Anspruch, an der weiteren Entwicklung der Weltbank aktiv mitzuwirken und unsere bilaterale Erfahrung in die Bank einfliessen zu lassen. Ebenso möchten wir die Stärken der Bank für die bilaterale Zusammenarbeit nutzen. Es ergeben sich dabei durchaus komplementäre Synergien. Die bisherige konstruktive Zusammenarbeit hat auch gezeigt, dass die Schweiz keine Berührungsängste vor der Grösse der Bank haben muss. Längerfristig ist es wichtig, dass sich die Schweiz aus einer gewissen selbstgewählten Isolation befreit hat und ihre entwicklungspolitische Stimme nun auch in einem grösseren Rahmen zum Tragen bringen kann. Dies ist mit dem Auftrag verbunden, in der Bank eine aktive Rolle zu spielen und darüber einen Dialog mit schweizerischen Entwicklungsorganisationen zu führen. Hier liegt eine wichtige Rolle von BAWI und DEH, nämlich eine Mittlerrolle zwischen einer zunehmend offenen und auch selbstkritischen Weltbank und den vermehrt auch für makroökonomische Überlegungen offenen privaten Entwicklungsorganisationen zu übernehmen.

\section{Osthilfe und Entwicklungszusammenarbeit}

Anfangs der 90er Jahre hat die Schweiz begonnen, Unterstützungsmassnahmen für Ost- und Mitteleuropa aufzubauen. Die Frage, ob Entwicklungszusammenarbeit und Osthilfe mehr Gemeinsames oder mehr Trennendes aufweisen, ist schon oft diskutiert worden. Aus der Sicht der Entwicklungspolitik ist die Antwort eindeutig: Entwicklungsfragen stellen sich im Osten ähnlich wie im Süden, und letztlich auch im Norden. Grosse Entwicklungsagenturen wie die Weltbank und viele bilaterale Geber haben daraus die organisatorischen Konsequenzen gezogen, indem sie aus den bestehenden Entwicklungszusammenarbeits-Strukturen heraus (und beruhend auf den vorhandenen Kenntnissen und Erfahrungen) die Osthilfe aufbauten. Diese Lösungen scheinen sich aufgrund erster Erfahrungen zu bewähren. Insbesondere auch deshalb, weil bisherige Zuordnungen zu Kategorien, wie "Dritte Welt" oder "Entwicklungsländer", ihre Bedeutung verloren und 
insbesondere auch nur noch bedingte Aussagekraft haben.

In der Schweiz sind für die Osthilfe und die Entwicklungszusammenarbeit sowohl im EDA wie im EVD verschiedene Dienststellen zuständig. Diese Aufteilung erscheint aus entwicklungspolitischer und operationeller Sicht nicht optimal, da wichtige fachliche, politische, operationelle und administrative Synergien nicht immer optimal genutzt werden können. Die humanitäre Hilfe in der DEH, mit den beiden Instrumenten der Humanitären Zusammenarbeit (HZA) und des Schweizerischen Katastrophenhilfekorps (SKH), ist zudem weltweit zuständig (und damit auch für Osteuropa), womit sich in diesem Bereich Überschneidungen ergeben. Wie problematisch die Angelegenheit ist, zeigt sich darin, dass - nach diesen Kriterien - Länder wie Tunesien oder Algerien in die Kategorie der Entwicklungsländer gehören, während etwa die zentralasiatischen Republiken nicht darunter fallen. Die bisherigen, künstlichen Kriterien der Zuteilung in die eine oder andere Gruppe müssten deshalb überprüft und überdacht werden. Aber auch von der operationellen Seite her (unter der wir Konzepte, Zusammenarbeitspolitik, Planung und Programmierung sowie Durchführung, Monitoring und Evaluation verstehen) sprechen viele Gründe dafür, dass die angewandten Instrumente in der Ostund Südhilfe ähnlichen Charakter aufweisen. Im Moment wird die organisatorische Eingliederung der Osthilfe im EDA überprüft. Es ist deshalb denkbar, dass in Zukunft andere organisatorische Lösungen gesucht werden.

In Bezug auf die finanziellen Mittel wird zwar immer wieder erklärt, dass die Osthilfe nicht auf Kosten der Südhilfe auf- und ausgebaut werde. Direkt und indirekt ist jedoch bei einem steigenden Budgetdefizit in den öffentlichen Haushalten ein Konkurrenzverhältnis zwischen den beiden Budgets vorhanden. Wie sich dies mittel- und langfristig auswirken wird, ist schwierig vorauszusehen. Das Auseinanderbrechen vieler Staatsstrukturen im Osten in immer kleinere, ethnisch-nationale Teilbereiche und die damit verbundenen kriegerischen Auseinandersetzungen führen zu Verunsicherungen und zu Auswirkungen in der Schweiz. Furcht vor zunehmenden Migrationen und Asylsuchenden sind die Folge und führen zum Ruf nach einer Reorientierung der Hilfe. Das politische Interesse ist vermehrt nach dem Osten gerichtet und dies nicht zuletzt wegen der geografischen Nähe.

Innen- und Aussenpolitik werden sich in Zukunft immer weniger klar voneinander abgrenzen lassen wie in früheren Jahrzehnten. Sie fliessen immer mehr ineinander über. Was heute irgendwo in der Welt geschieht, hat morgen auch Auswirkungen auf die Schweiz. Deshalb werden die allgemein langfristige Entwicklungszusammenarbeit, die kurzfristige humanitäre Hilfe sowie die Osthilfe in Zukunft stärker auch unter innenpolitischen Blickwinkeln gesehen.

In diesem Zusammenhang sind auch die Anstrengungen der DEH zu sehen, sich vermehrt mit Mittelmeerfragen zu befassen (Errichtung einer Stabsstelle) und insbesondere die Beziehungen zur europäischen Gemeinschaft auch im Bereich der europäischen Entwicklungspolitik zu verstärken, die Koordination und die Zusammenarbeit zu verbessern und einzelne Massnahmen aufeinander abzustimmen. Dies zeigte sich kürzlich auch bei der Palästinahilfe, in der die Schweiz aktiv mitwirkt und in engster Koordination mit den europäischen Staaten und internationalen Institutionen, wie Weltbank und UNDP, vorgeht. 


\section{Rio 1992 und die bilaterale Entwicklungszusammenarbeit}

Unter den neuen Tendenzen seit Beginn der 90er Jahre nehmen Umweltfragen einen zunehmend wichtigeren Einfluss auf die Ausrichtung der bilateralen Entwicklungszusammenarbeit der Schweiz ein. Mit der Umweltproblematik hat sich die DEH allerdings schon früher eingehend befasst und Nachdruck auf umweltschonende Unterstützungsmassnahmen gelegt. Auch in Entwicklungsländern nimmt die Einsicht zu, dass sie durch die Zerstörung ihrer Umwelt ihre eigenen Lebensgrundlagen untergraben und ihre Entwicklungsmöglichkeiten damit langfristig immer mehr einschränken. Nachhaltigkeit ist deshalb heute in der entwicklungspolitischen Diskussion zu einem zentralen und anerkannten Grundsatz geworden. Langfristige Entwicklung kann nur bei gleichzeitigem Erhalt der natürlichen Ressourcen erreicht werden. Allerdings sind damit noch keine Probleme gelöst. Erhaltung der Umwelt, Entwicklung, Produktionswachstum und Armutsbekämpfung stehen heute im Zentrum des internationalen entwicklungspolitischen Interesses, aber sie sind nicht konfliktfrei. Unter dem Druck, ihre Bevölkerung zu ernähren, mangels angepasster Langfriststrategien und auch in Anbetracht ihrer schwierigen wirtschaftlichen und finanziellen Situation haben Entwicklungsländer oft kurzfristig keine andere Wahl als ihr Überleben durch eine weitere Übernutzung ihrer natürlichen Ressourcen zu sichern.

Im Umweltbereich sind in der schweizerischen Enwicklungszusammenarbeit in den letzten Jahren besondere Akzente gesetzt worden. Die Botschaft über die Weiterführung der technischen Zusammenarbeit und der Finanzhilfe zugunsten von Entwicklungsländern vom 21. Februar 1990 geht ausführlich auf die Umweltproblematik und insbesondere auch auf die Verknüpfung von Umwelt und Armut ein.

Einen besonderen Akzent setzte dann die von den schweizerischen Hilfswerken lancierte Petition "Entwicklung braucht Entschuldung", die 1991 zum Entschuldungs- und Umweltrahmenkredit von 700 Millionen Franken führte, von denen 300 Millionen Franken für bilaterale und multilaterale globale Umweltprogramme bestimmt sind. Dieses klare politische Zeichen hat nicht nur das Engagement der Schweiz im globalen Umweltbereich verstärkt, sondern auch im übrigen bilateralen Bereich das Bewusstsein für Umweltfragen gestärkt.

Schliesslich hat im Juni 1992 der Erdgipfel von Rio (UNCED) mit der "Agenda 21" ein eigentliches Umwelt-Arbeitsprogramm für das 21. Jahrhundert ausgearbeitet. Darin wird versucht, eine gesunde Umwelt, Wirtschaftswachstum und Armutsbekämpfung für die Menschen dieser Erde miteinander in Einklang zu bringen. Es stellt eine eigentliche Überlebenscharta für das Raumschiff Erde dar. Als direkte Folge davon ist unter dem Stichwort "Suivi de Rio" (d.h. dem Umsetzungsprogramm von Rio) ein interdepartementales Komitee in der Bundesverwaltung gegründet worden ("CIRio"), in dem die DEH zusammen mit BUWAL und BAWI eine leitende Rolle wahrnimmt. Die DEH hat dabei vor allem die Aufgabe, Interessen der Entwicklungsländer in die Überlegungen bei neuen Vorlagen und Gesetzen einzubringen, in den verschiedenen Arbeitsgruppen die "Südsicht" zu 
vertreten und einen "Aktionsplan der Schweiz für eine nachhaltige Entwicklung" zu fördern.

Die Konferenz von Rio hat aber auch die operationellen Programme beeinflusst. Ein besonderer Akzent wird auf die Integration der Umweltaspekte in die Sektor- und Landesprogramme der DEH gelegt. Zusätzlich sind eine Reihe von Prioritäten für die Unterstützungsmassnahmen der DEH formuliert worden:

- Die Verstärkung der lokalen Kapazitäten zur Durchführung von Umweltprogrammen in Entwicklungsländern;

- die Verstärkung der Aktivitäten im Bereich der Bergregionen, insbesondere im Bereich der regionalen Kooperation zwischen Ländern mit ähnlichen ökologischen Fragestellungen;

- die Unterstützung einer nachhaltigen Nutzung und Erhaltung der Tropenwälder;

- die Bekämpfung der zunehmenden Verwüstung und die Abnahme der Bodenfruchtbarkeit;

- die Verstärkung der Aktivitäten im Bereich des integrierten TrinkwasserManagements (Wasserversorgung, Schutz der Oberflächengewässer, Wasserhygiene, Abwasser etc.);

- die Unterstützung bei der Abfallbeseitigung und -entsorgung.

Im Politikbereich der DEH wurde eine "Sektorpolitik Umwelt" formuliert, und ein mittelfristiges Umweltprogramm wird erarbeitet.

Diese Prioritäten gelten sowohl für den multilateralen wie für den bilateralen Bereich. In den kommenden Jahren wird es darum gehen, diese Schwerpunkte im bilateralen Programm noch verstärkt zu verankern. Die Rio-Konferenz hat somit der bilateralen EZA der DEH starke neue Impulse in Richtung nachhaltige Entwicklung gegeben. Gleichzeitig hat sie auch die Sensibilität erhöht für die Problematik von Entwicklung, Umwelt und Armut, für Zielkonflikte zwischen Produktionssteigerung und Umweltmassnahmen sowie für die begrenzte Belastbarkeit von Systemen.

\section{Auswirkungen dieser neuen Tendenzen auf die bilaterale schweizerische Entwicklungszusammenarbeit}

Welches sind die Auswirkungen dieser verschiedenen neuen Tendenzen auf die bilaterale schweizerische Entwicklungszusammenarbeit? Diese Zusammenarbeit ist komplexer, anforderungsreicher und schwieriger geworden. Fachliche, persönliche, entwicklungspolitische und konzeptionelle Anforderungen nehmen zu. Die zunehmende "Professionalität" führt zu veränderten Anforderungsprofilen der Mitarbeiterinnen und Mitarbeiter. Der rasche Wandel verlangt Anpassungsfähigkeit und klare Prioritäten für die Entwicklungszusammenarbeit:

- Angepasste Unterstützungsmassnahmen setzen gute, langjährige und breitgefächerte Kenntnisse und Erfahrungen der lokalen, regionalen und nationalen 
Gegebenheiten eines Landes voraus, sowohl im sozialen wie auch im wirtschaftlichen, politischen, gesellschaftlichen, institutionellen und geografischen Bereich, um nur einige zu nennen. Misserfolge in der Entwicklungszusammenarbeit sind oft auf mangelnde Kenntnisse des "lokalen Umfeldes" und der wirklichen Interessen der von einer Unterstützungsmassnahme Betroffenen zurückzuführen. Nur der längerfristige und vertiefte Blick auf die oft vielschichtige Realität hinter einer sachlich formulierten Programmproblematik führt zu angepassten Lösungen. Dies erfordert eine geografische Konzentration unserer Unterstützung.

- Fachliche Kenntnisse und Schwerpunkte sind eine zweite wichtige Voraussetzung für erfolgversprechende Programme. Gerade weil unsere westlichen Kenntnisse mit lokalen Fachkenntnissen verbunden und unsere Konzepte entsprechend angepasst werden müssen, ist eine laufende Beschäftigung und Auseinandersetzung mit neuen Fachkonzepten und -erfahrungen notwendig.

- Nicht nur geografisch und sektoriell, sondern auch vom Unterstützungsansatz her ist eine Konzentration notwendig. Schlüsselworte sind hier Förderung der Selbsthilfe und aktive Partizipation als Unterstützungsphilosophie. Damit wollen wir eine von der Bevölkerung getragene Entwicklung, da nur mit einer starken Verankerung bei den Nutzniessern ein Programm nachhaltige Wirkung haben wird.

- Im internationalen Bereich - in der Diskussion, insbesondere mit anderen Gebern - werden wir dort ernst genommen, wo wir aufgrund von Schwerpunkten konkrete Kenntnisse haben, die wir in die Diskussion einbringen und zur Verfügung stellen können. Geografische und fachliche Schwerpunkte sind eine Voraussetzung für eine bedeutsame Rolle in der internationalen Koordination und im internationalen Geberdialog; d.h. auch in der Möglichkeit, die Massnahmen anderer Geber nachhaltig zu beeinflussen.

- Verstärken wird sich der längerfristige Politikdialog mit Entwicklungsländern, wobei für uns praktische, konkrete Erfahrungen mit Programmen eine Voraussetzung sind, um Änderungen in der Politik oder in einem Bereich (z.B. Landwirtschaft, Gesundheit, Berufsbildung) nicht nur theoretisch, sondern auch praktisch abzustützen.

- Auf der Projekt- und Programmebene wird sich die Verlagerung vom Projekt zum Programm, insbesondere zum Sektorprogramm (z.B. Gesundheit, Forstwirtschaft, Viehzucht, nachhaltige Landnutzung, Demokratisierung, Dezentralisierung etc.) verstärken. Diese Tendenz geht einher mit der stärkeren Konzentration und Professionalisierung unserer Tätigkeit.

- Das isolierte Einzelprojekt oder -programm wird abgelöst durch Landes- und Sektorprogramme; die technische und Finanzhilfe ergänzt durch Sektorprogramm- und Politikdiskussionen.

- Die Zahl des langfristig eingesetzten ausländischen Feldpersonals wird weiterhin zurückgehen und wird durch lokale Fachleute sowie kurzfristig eingesetzte Berater ersetzt.

- Nur mit Schwerpunkten ist es uns möglich, unsere Professionalität als EZAOrganisation bei knappen personellen und finanziellen Ressourcen zu verstär- 
ken. Sie erlaubt eine langfristige Personal- und Laufbahnplanung und damit die Heranbildung eines professionellen Kaders. Dies gilt nicht nur für die DEH, sondern im weiteren Sinn auch für Entwicklungsorganisationen, Unternehmungen und andere Institutionen, die mit ihr zusammenarbeiten.

Diese Gründe für eine klare Schwerpunktorientierung werden durch die Budgetrestriktionen noch verstärkt und sind entscheidend für die Qualität unserer zukünftigen Unterstützungsmassnahmen. Landes- und Jahresprogramme der Schwerpunktländer sowie Sektorpolitiken und -programme sind wichtige Instrumente für ihre Umsetzung. Allerdings ist diese Schwerpunktpolitik für eine breite Öffentlichkeit nicht immer leicht verständlich. Ebenso wird sie durch Anträge um Projektfinanzierungen von verschiedensten Seiten und mit verschiedenen Interessen immer wieder in Frage gestellt. Sie muss deshalb erklärt und die Gründe dafür müssen dargelegt werden.

Verstärken werden sich in Zukunft - gerade wegen der Sparmassnahmen und vor allem in den bilateralen Programmen - auch die Querschnitthemen. Es handelt sich dabei um Anliegen, die in allen Programmen zum Tragen kommen müssen und auf die bei der Identifikation, der Vorbereitung und der Planung sowie der Durchführung und der Evaluation besonderes Gewicht gelegt wird. Gerade weil nur beschränkte finanzielle Mittel vorhanden sind, können neue Konzepte, Ideen und Bedürfnisse in erster Linie über die Verstärkung solcher Komponenten in bestehenden Programmen verwirklicht werden. Prioritäre Querschnitthemen in unseren bilateralen Programmen in den kommenden Jahren sind:

- die Frauenförderung,

- die Good Governance,

- die Nachhaltigkeitsfragen,

- die Partizipation als Grundsatz,

- die Förderung und Stärkung von Institutionen,

- die Umweltauswirkungen.

\section{Das EZA-Kooperationssystem der Schweiz oder mit wem arbeitet die DEH in der Schweiz zusammen?}

Institutionell ist die EZA der Schweiz seit Beginn durch eine grosse Pluralität gekennzeichnet. DEH und private Entwicklungshilfeorganisationen, aber auch andere Träger, wie die Privatwirtschaft, arbeiten seit langem eng zusammen, wobei sich immer mehr komplementäre Aufgaben ergeben. Welchen Einfluss haben die neuen Tendenzen auf die verschiedenen Institutionen in der schweizerischen Entwicklungszusammenarbeit?

Auf der Politikebene, insbesondere im innenpolitischen Bereich, ist trotz weiterer Sparmassnahmen im Budgetbereich auf eine erhöhte Solidarität zwischen grossen Hilfswerken und der DEH zu hoffen. Es geht darum, als gemein- 
same Aufgabe das Niveau der gesamten öffentlichen Leistungen der Schweiz im Entwicklungsbereich zu halten und möglichst rasch auf das vom Bundesrat erklärte Ziel von $0,4 \%$ des Bruttosozialprodukts zu erhöhen. Von Budgetkürzungen werden sowohl Hilfswerke wie auch DEH betroffen sein, und es liegt in beiderseitigem Interesse, Budgetabstriche möglichst klein zu halten. Eine wichtige Komponente im innenpolitischen Bereich ist die gemeinsame Sorge um eine breitere und vertiefte Akzeptanz der Entwicklungszusammenarbeit und der Nord-Süd-Probleme bei einem breiten Teil der Öffentlichkeit. Bund und Hilfswerke haben hier eine gemeinsame Aufgabe, die sie komplementär, jeder mit seinen Mitteln, lösen können. Diese politische und vor allem innenpolitische Sicht wird an Bedeutung gewinnen. Es ist für das Verständnis der Beziehungen zwischen Nord und Süd wichtig, dass uns vermehrt bewusst wird, dass z.B. unsere Landwirtschaftspolitik, unsere Gesetzgebung im Bereich von Biotechnologie und Gentechnik oder unsere Energiepolitik direkt etwas mit dem Süden zu tun haben.

Im entwicklungspolitischen Bereich dürfte sich zwischen Bund und Hilfswerken die bisherige gesunde Spannung erhalten, die immer wieder auch zu Diskussionen, zu teilweise verschiedenen Standpunkten und zu einem fruchtbaren Dialog führt. Die gegenseitigen Unterschiede scheinen über die Jahrzehnte jedoch eher kleiner geworden, und der Dialog zwischen Verwaltung und Hilfswerken ist offener und auch ergiebiger. Die Bereitschaft zum Zuhören und die Möglichkeiten zur Diskussion werden nicht nur vermehrt angeboten, sondern auf beiden Seiten auch vermehrt genutzt. Auch in Zukunft wird diese Diskussion weiter vertieft. Die DEH ist überzeugt, dass diese Diskussion und Auseinandersetzung für beide Teile notwendig und wichtig ist.

Im Programmbereich (Mitfinanzierung von Programmen der grossen Entwicklungshilfeorganisationen durch die DEH) sind durch die Einführung des Programmbeitrags neue Wege in der Zusammenarbeit begangen worden. Hier wird sich in Zukunft das Gewicht der Diskussion noch vermehrt auf die grundsätzliche entwicklungspolitische Ausrichtung der Programme, auf konzeptionelle Fragen im operationellen Bereich sowie auf die Langfristausrichtung dieser Programme in Bezug auf geografische, sektorielle, institutionelle und methodische Schwerpunkte richten. Vermehrt gefördert werden sollten die Ländergruppen, d.h. die Zusammenkunft aller in einem Land tätigen Hilfsorganisationen zum regelmässigen Erfahrungs- und Informationsaustausch.

Im Regiebereich schliesslich (d.h., im Auftragsbereich der DEH) dürfte sich eine stärkere Komplementarität der Zusammenarbeit ergeben mit einer verstärkten Spezialisierung und einer längerfristigen Absprache über gegenseitige Prioritäten. Gleichzeitig zeichnet sich bei Hilfswerken - aufgrund der Budgetprobleme - die Tendenz ab, neue Geldquellen und Auftraggeber zu finden. Ein vor kurzem stattgefundenes Seminar zwischen den wichtigsten Regieorganisationen und der DEH hat hier die Grundsätze der Zusammenarbeit festgelegt. Damit ist ein wichtiger Schritt im gegenseitigen Rollenverständnis erreicht worden.

Modellhaften Charakter hat die Zusammenarbeit im Entschuldungsbereich zwischen BAWI/DEH einerseits und der Arbeitsgemeinschaft der Hilfswerke andererseits; mit dieser innovativen Zusammenarbeit zeichnen sich echte kom- 
plementäre Synergien zwischen verschiedenen EZA-Trägern ab, die für die Zukunft Vorbildcharakter haben.

Die vorangehenden Gedanken und Überlegungen sind aus der Situation im Herbst 1993 entstanden. In einer Zeit des Wandels über neue Tendenzen zu schreiben, ist jedoch nicht ohne Risiken. In diesem Sinn soll dieser Beitrag auch verstanden werden, nämlich als zukünttige, mögliche Entwicklungen in einem weiterhin sich rasch ändernden Umfeld.

\section{Résumé}

Au cours de ces dernières années, la coopération bilatérale de la Suisse avec les pays en développement a été affectée d'une part par la transformation de l'environnement international et d'autre part par des changements intervenus sur le plan national. Cet article analyse ces changements (la fin de la guerre froide et la coopération avec les pays de l'Est, les conséquences de la Conférence de Rio sur l'environnement et le développement, l'adhésion de la Suisse aux institutions de Bretton Woods et les restrictions budgétaires résultant de la situation financière de la Confédération. La conclusion de cette analyse est notamment que la coopération au développement devient une tâche de plus en plus complexe qui requiert plus de professionnalisme ainsi que la fixation plus marquée de priorités. Les programmes sectoriels prendront de plus en plus le pas sur les projets et programmes isolés. 